\title{
Anti-reflection coated spectrally selective carbon nanotube solar absorbers
}

\author{
Zhonghua Chen ${ }^{1, *}$ and Tobias Boström ${ }^{2}$ \\ ${ }^{1}$ Norut Northern Research Institute Narvik AS, 8517, Narvik, Norway \\ ${ }^{2}$ UiT, the Arctic University of Norway, Department of Physics and Technology, 9019, Troms $\varnothing$, Norway
}

\begin{abstract}
Multi-walled carbon nanotube (MWCNT) solar absorbers on aluminium substrates have been prepared by electrophoresis. The MWCNT absorbers exhibit a good spectral selectivity over the visible and infrared wavelengths. However, to further enhance the solar absorption, an anti-reflection coating made from porous silica was spin-coated on top of MWCNT solar absorbers. Porous silica precursor was prepared using tetraethyl orthosilicate (TEOS), and Pluronic F127 was used as a pore-forming agent. A maximum solar absorptance gain of 0.02 was achieved, which improves the spectral selectivity of MWCNT absorbers. The effect of anti-reflection layer depends on not only its thickness/refractive index but also the roughness of aluminium substrate.
\end{abstract}

\section{Introduction}

The solar thermal absorber is the key component of a solar thermal collector. Highly effective solar thermal absorbers should have a low reflectance in the UV/Vis/NIR region of solar spectrum and a high reflectance at wavelengths $>2.5 \mu \mathrm{m}$. Hence, they are called spectrally selective solar absorbers (SSSA). A tandem structure [1] consisting of an absorbing layer and a reflective substrate is usually used. Aluminium plates $[2,3]$ are often used as the substrates owing to its high reflectance $(>95 \%)$ in the infrared region [4], lower density and lower price compared to other metals. Cermet films referred to as metaldielectric composites are well known as SSSAs. However, the fabrication of such coatings is normally complicated and expensive, and requires for example vacuum depositing methods like sputtering, physical or chemical vapor deposition. The objective of this work is to create highly selective SSSAs but using inexpensive and simple methods. We have used multi-walled carbon nanotubes (MWCNT) in the absorbing layer, which simply can be coated on top of an aluminium substrate by electrophoretic deposition (EPD). Simulations using single-walled carbon nanotubes (SWCNT) as absorbing layers was done by the authors previously, and indicated that they can be good candidates as SSSA owing to their high absorption over the solar spectrum [5]. In addition, carbon nanotubes have excellent stability against heat, water, oxidizing environments and standard chemicals.

\footnotetext{
* e-mail: zhonghua.chen@norut.no
}

To reduce the optical loss, i.e. increase solar absorption of solar absorbers, anti-reflective coatings (ARC) can be applied on top of them $[6,7]$.

\section{Method \\ 2.1 Anti-reflection theory}

To be the most effective, the anti-reflective layer should have a refractive index equalling to the square root of the refractive index of the underlying layer. In addition, the ARC thickness has to be selected carefully so that the destructive interference effect of the ARC suppresses the reflectance at the most suitable wavelength. This wavelength normally equals to the wavelength where the underlying layer generates its first constructive interference maximum, which in our case is between 600 and $800 \mathrm{~nm}$, see Figure 1a.

The prepared MWCNT coating for anti-reflection experiments had a real part of refractive index of 1.38 at $550 \mathrm{~nm}$, evaluated by Spectroscopic Ellipsometry. The Ellipsometry measurements were challenging on the relatively rough CNT samples and hence, the uncertainty of the results is high. The most effective ARC for MWCNT absorbers should be the square root of 1.38 , which equals to 1.17. Unfortunately, there are no solid materials with such a low refractive index. Thus, porous silica (PS) whose refractive index could be tuned by changing the porosity $[8,9]$ was employed on MWCNT absorbers as the ARC in this report.

\subsection{Sample preparation}

All chemicals were used as received without any pretreatments. The preparation procedure of MWCNT solar absorber samples has been reported in the authors' previous 


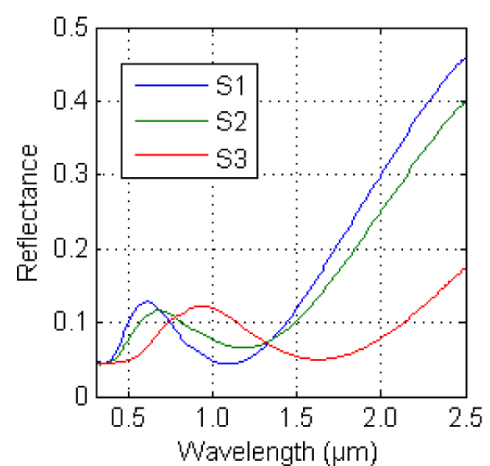

a

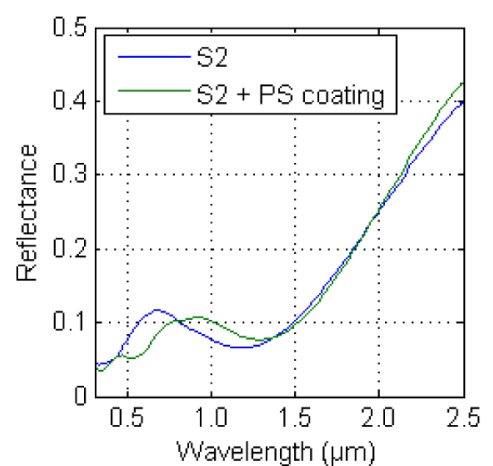

b

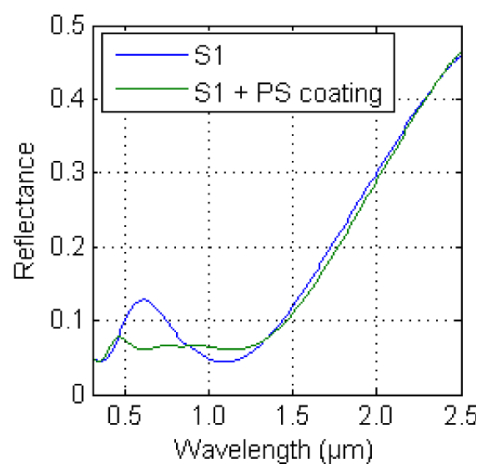

c

Fig. 1. Reflectance spectra of multi-walled carbon nanotube (MWCNT) absorbers: (a) deposited with various thicknesses of MWCNT layers; (b) and (c) coated with porous silica layer.

work [10], where functionalized MWCNTs (Nanocyl, Belgium) were dispersed and stabilized in aqueous solution with the addition of an ionic surfactant. The stable MWCNT suspension was applied for EPD on aluminium substrates (Oudifu, China). Obtained MWCNT coatings were dried in room conditions and heat-treated.

The sol-gel method was employed to produce PS precursor using tetraethyl orthosilicate (TEOS) as starting material. TEOS (ACROS Organics) was mixed with ethanol (Merck) before DI water was added to the solution. Hydrochloric acid ( $\mathrm{HCl}$, Merck) was used as hydrolyzing catalyst. After stirring for half an hour, the silica sol was added in a pore-forming agent Pluoric ${ }^{\circledR} \mathrm{F} 127$ (PF127, Sigma-Aldrich). Allowing hydrolysis for two hours, the silica sol containing a TEOS: ethanol: $\mathrm{H}_{2} \mathrm{O}$ : PF127 molar ratio of 1: 50: 15: 0.008 was ready for coating process.

A spin coater Specialty Coating Systems, model 6808) was employed for forming silica films on top of MWCNT solar absorbers. The spin speed and duration were tuned for adjusting the thickness of silica coatings. Lastly, the coatings were dried at $80^{\circ} \mathrm{C}$ for half an hour and heat treated at $450{ }^{\circ} \mathrm{C}$ for 45 minutes in air in a tube oven. After cooling to room temperature, the samples were submitted to reflectance measurement.

\subsection{Characterization techniques}

A solar thermal absorber is usually evaluated by two parameters: solar absorptance and thermal emittance under a normal angle of incident radiation. Normal solar absorptance, $\alpha$, is theoretically defined as a weighted fraction of the absorbed radiation to the incoming solar radiation on a surface, as shown in equation (1). The spectral solar irradiance, $I_{\text {sol }}$, is defined according to ISO standard 9845-1 (1992) for air mass of 1.5. $\lambda$ is the wavelength of incident radiation in the unit of $\mu \mathrm{m}$ and $R(\lambda)$ is the reflectance at a certain wavelength.

$$
\alpha=\frac{\int_{0.3}^{2.5} I_{\mathrm{sol}}(\lambda)(1-R(\lambda)) d \lambda}{\int_{0.3}^{2.5} I_{\mathrm{sol}}(\lambda) d \lambda} .
$$

Normal thermal emittance, $\varepsilon$, is the ratio of emitted radiation of a surface to that of Plank's blackbody, $I_{\mathrm{p}}$, at $100{ }^{\circ} \mathrm{C}$ and can be calculated following equation (2):

$$
\varepsilon=\frac{\int_{2.0}^{50} I_{p}(\lambda)(1-R(\lambda)) d \lambda}{\int_{2.0}^{50} I_{p}(\lambda) d \lambda} .
$$

The normal reflectance of prepared solar absorber samples was measured in the wavelength interval of 0.3-20 $\mu \mathrm{m}$. A Perkin-Elmer Lambda 950 spectrophotometer, equipped with an integrating sphere of diameter $150 \mathrm{~mm}$, circular beam entrance and sample port of $25 \mathrm{~mm}$, was used in the wavelength interval $0.3-2.5 \mu \mathrm{m}$. The infrared wavelength range, 2.5-20 $\mu \mathrm{m}$, was covered with a Bruker Tensor FT-IR spectrophotometer. The reflectance data between 20 to $50 \mu \mathrm{m}$ was extrapolated by a defined algorithm.

There are different ways of calculating spectral selectivity using solar absorptance and thermal emittance. The ratio of solar absorptance to thermal emittance $(\alpha / \varepsilon)$ is one of often used methods. However, we judge this method as inappropriate. For example, a solar absorber with a solar absorptance of 0.60 and a thermal emittance of 0.04 equalling to a ratio of 15 does not have higher photothermal conversion efficiency than a solar absorber with a solar absorptance of 0.90 and a thermal emittance of 0.1 , which achieves the ratio of only 9 . The opposite is actually true since solar absorptance is twice as important as thermal emittance, referred to the newly issued ISO standard about absorber surface durability [11]. In this paper, we calculate spectral selectivity using the expression $\alpha-0.5 \varepsilon$, which reflects the weight factor of thermal emittance in a more reasonable matter.

A Veeco NT9080 Profilometer, using high precision white light interferometry was employed to estimate the thickness of the MWCNT coatings.

\section{Results and discussion}

By tuning the deposition time during EPD, while keeping the other EPD parameters like applied voltage and the interelectrode spacing unchanged, various thicknesses of MWCNT coatings can be formed on the aluminium substrates. 
Reflectance spectra for solar absorbers with different thicknesses of MWCNT coatings are displayed in Figure 1a. From samples S1, S2 to S3, the thickness of MWCNT coating increases. As we can see from the curves, the peak reflectance as a result of thin film interference moves to longer wavelength with thicker MWCNT coating. Compared to samples S1 and S2, sample S3 has a higher solar absorptance due to the lower reflectance at around $550 \mathrm{~nm}$ where the solar spectrum has a maximum intensity. However, with thicker absorbing layer, a larger part of the emitted infrared light, i.e. heat, is lost to the surroundings. This results in a higher thermal emittance, the overall spectral selectivity is even lower.

Figures $1 b$ and $1 c$ show the reflectance data of two PS coated MWCNT absorbers. For sample S2 (Fig. 1b), the PS coating results in a reduction in reflectance from 0.3 and $0.9 \mu \mathrm{m}$. However, the destructive interference effect of the $\mathrm{ARC}$ is misplaced, it is at $520 \mathrm{~nm}$ when it should have been at $690 \mathrm{~nm}$ to have the greatest effect. Hence, the gain in solar absorptance is only 0.01 . The thermal emittance after PS coating for sample 4 is 0.19 . As shown in Figure 1c, sample S1 has a well-placed destructive interference dip at $600 \mathrm{~nm}$, corresponding to the peak reflectance of the absorber layer. After PS coating, an interference minima was achieved. As a result, the peak reflectance was largely suppressed. The calculated gain in solar absorptance was 0.02 increased from 0.90 to 0.92 . The thermal emittance after PS coating for sample $\mathrm{S} 1$ is 0.10 . The calculated spectral selectivity is 0.87 .

The increase of solar absorption by adding AR coating is not as high as expected. The minimum reflectance after AR coating is still around $5 \%$, when it should be close to zero. This is probably due to a mismatch in refractive indices, i.e. the refractive index of PS layer is not the square root of the refractive index of the MWCNT coating. Searching for an ARC with a more suitable refractive index will be addressed in further work. There is also another possible reason for the fairly small increase in solar absorptance after PS coating, which is because coating, and/or substrate inhomogeneity. If the substrate or the coatings are not well defined and homogenous, then the interference effect will be reduced, resulting in a smaller solar absorptance gain than expected. The homogeneity issue will as well be addressed in future work.

\section{Conclusions}

PS layer coated on top of MWCNT layers enhances the solar absorption of MWCNT absorbers by depressing the peak reflectance. The gain of solar absorptance was not much as expected due to either the mismatch of refractive indices between the PS coating and the MWCNT layer and/or the inhomogeneity of coating and substrate. Future work will address the refractive index and inhomogeneity issues and evaluate the long-term durability of the MWCNT absorbers.

This work was supported by Nano2021 program of the research council of Norway and the company ASV Solar AS.

We would like to express gratitude to Thomas Wagner from LOT-Quantum Design GmbH for technical support on Ellipsometry.

\section{References}

1. O.P. Agnihotri, B.K. Gupta, Solar selective surfaces (John Wiley \& Sons, New York, 1981)

2. T. Boström, G. Westin, E. Wäckelgård, Sol. Energy 74, 497 (2003)

3. P. Konttinen, T. Salo, P.D. Lund, Sol. Energy 78, 41 (2005)

4. J. Bartl, M. Baranek, Meas. Sci. Rev. 4, section 332 (2004)

5. Z. Chen, A. Jain, T. Boström, Energy Proc. 58, 179 (2014)

6. T. Boström, Solution-chemically derived spectrally selective solar absorbers (Uppsala University, Uppsala, 2006)

7. T. Boström, E. Wäckelgård, G. Westin, Sol. Energy Mater. Sol. Cells 84, 183 (2004)

8. P. Karasinski, J. Jaglarz, M. Reben, E. Skoczek, J. Mazur, Opt. Mater. 33, 1989 (2011)

9. R. Prado, G. Beobide, A. Marcaide, J. Goikoetxea, A. Aranzabe, Sol. Energy Mater. Sol. Cells 94, 1081 (2010)

10. Z. Chen, T. Boström, Sol. Energy Mater. Sol. Cells 144, 678 (2016)

11. ISO 22975-3, Solar energy-collector components and materials - Part 3: absorber surface durability (International Organization for Standardization, Switzerland, 2014)

Cite this article as: Zhonghua Chen, Tobias Boström, Anti-reflection coated spectrally selective carbon nanotube solar absorbers, Renew. Energy Environ. Sustain. 1, 2 (2016) 